\title{
Total Laparoscopic Partial Hepatectomy Versus Open Partial Hepatectomy For Primary Left-Sided Hepatolithiasis: Study Protocol For A Randomized Controlled Trial
}

\author{
Shu-bo Pan \\ Second Affiliated Hospital of Anhui Medical University \\ Chun-li Wu \\ Second Affiliated Hospital of Anhui Medical University \\ Da-chen Zhou \\ Second Affiliated Hospital of Anhui Medical University \\ Qi-ru Xiong \\ Second Affiliated Hospital of Anhui Medical University \\ Xiao-ping Geng \\ Second Affiliated Hospital of Anhui Medical University \\ Hui Hou ( $\nabla$ hui0402@hotmail.com )
}

Second Affiliated Hospital of Anhui Medical University https://orcid.org/0000-0002-5568-4644

Study protocol

Keywords: Laparoscopic, open liver resection, ERAS, Hepatectomy

Posted Date: July 19th, 2021

DOI: https://doi.org/10.21203/rs.3.rs-17346/v2

License: (c) (i) This work is licensed under a Creative Commons Attribution 4.0 International License. Read Full License 


\section{Abstract}

Background: The advantages of laparoscopic left-sided hepatectomy (LLH) for treating hepatolithiasis in terms of the time to postoperative length of hospital stay (LOS), morbidity, long-term abdominal wall hernias, hospital costs, residual stone rate and recurrence of calculus have not been confirmed by a randomized controlled trial. The aim of this trial is to compare the safety and effectiveness of LLH to those of open left-sided hepatectomy $(\mathrm{OLH})$ for the treatment of hepatolithiasis.

Methods: Patients with hepatolithiasis eligible for left-sided hepatectomy will be recruited. The experimental design will produce two randomized arms (laparoscopic and open hepatectomy) at a 1:1 ratio and a prospective registry. All patients will undergo surgery in the setting of an enhanced recovery after surgery (ERAS) program. The prospective registry will be based on patients who cannot be randomized because of the explicit treatment preference of the patient or surgeon or because of ineligibility (not meeting the inclusion and exclusion criteria) for randomization in this trial. The primary outcome is the LOS. The secondary outcomes are percentage readmission, morbidity, mortality, hospital costs, long-term incidence of incisional hernias, residual stone rate and recurrence of calculus. It will be assumed that, in patients undergoing LLH, the length of hospital stay will be reduced by 1.3 days. A sample size of 44 patients in each randomization arm has been calculated as sufficient to detect a 1.3-day reduction in LOS ( $90 \%$ power and $a=0.05$ (two-tailed)). The trial is a randomized controlled trial that will provide evidence for the merits of laparoscopic surgery in patients undergoing liver resection within an ERAS program.

Discussion: Although the outcomes of LLH have been proven to be comparable to those of OLH in retrospective studies, the use of LLH remains restricted, partly due to the lack of short-and long-term informative RCTs pertaining to patients with hepatolithiasis in ERAS programs. To evaluate the surgical and long-term outcomes of LLH, we will perform a prospective RCT to compare LLH with OLH for hepatolithiasis within an ERAS program.

Trial registration: ClinicalTrials.gov NCT03958825. Registered on 21 May 2019.

\section{Background}

Hepatolithiasis is defined as a gallstone disease in the intrahepatic bile ducts and is mainly prevalent in Southeast Asia[1]. Hepatolithiasis may occur alone or with extrahepatic bile duct stones. Long-term hepatolithiasis can cause secondary biliary stricture, liver cirrhosis and even cholangiocarcinoma[2]. Hepatectomy is one of the most effective methods for treating intrahepatic stones because it can simultaneously remove stones and relieve bile duct stricture[3, 4]. For hepatectomy of hepatolithiasis, due to the presence of stones in the left liver of most patients with intrahepatic bile duct stones, left hepatectomy, including left lateral hepatectomy and left hepatectomy, is the most common procedure performed. With the advancement of laparoscopic technology, laparoscopic liver resection has been used in the treatment of various liver diseases, including benign and malignant liver tumors[5, 6]. Since Louisville's statement in 2008, the international status of laparoscopic hepatectomy has been widely recognized[7]. However, laparoscopic hepatectomy in patients with hepatolithiasis may be more difficult and challenging than open hepatectomy because patients with hepatolithiasis usually experience changes in their normal anatomy and perihepatic adhesion due to chronic inflammation[8-10]. To date, only a few retrospective studies have compared the outcomes of laparoscopic left-sided hepatectomy (LLH) with those of open leftsided hepatectomy (OLH) for hepatolithiasis, and the feasibility and efficacy of LLH have not been fully evaluated[11, 12].

Enhanced recovery after surgery (ERAS) programs have been applied for patients receiving small-scale and large-scale liver resection[13,14]. A number of studies have shown that ERAS programs are safe, feasible and effective in reducing the median LOS in both open and laparoscopic resection[15, 16]. In some retrospective trials comparing earlier recovery or a reduction in LOS, perioperative evaluation or management was not uniform[12, 17]. In addition, the value of laparoscopic leftsided hepatectomy for hepatolithiasis compared with that of open left-sided hepatectomy within an ERAS program in terms LOS and hospital costs has not been studied in a randomized controlled trial (RCT). However, randomized grouping of patients undergoing open or laparoscopic liver resection is dangerous. Due to the lack of clinical and patient equipoise of 
laparoscopic surgery, experienced centers and surgeons are reluctant to randomize patients. To take advantage of two centers' preference and disapproval of laparoscopic liver surgery to obtain a series of uninterrupted prospective patients, we constructed a randomized controlled trial design with two randomized groups (open and laparoscopic surgery) and a prospective registry. Combining an RCT with a prospective registry will improve the overall power and enhance the external validity and generalizability of the study results[18].

\section{Methods And Design}

A randomized clinical trial and a prospective registry with two parallel groups will be conducted at general surgery department of the Second Affiliated Hospital of Anhui Medical University in China. The aim of this study is to compare the effect of two hepatectomy methods for hepatolithiasis by assessing factors related to mortality and morbidity, including postoperative length of hospital stay, biliary leakage[19], post-hepatectomy hemorrhage[20], post-hepatectomy liver failure[21], incisional hernias, residual stone rate, and recurrence of calculus[22].

\section{Sample size}

The sample size calculation is based on the primary endpoint: the LOS. According to published data, an assumed a 1.3-day reduction in LOS is the appropriate basis for the calculation, assuming 4.5 days in the LLH group and 5.8 days in the OLH group[23]. This calculation yields a total of 40 patients in each group, which assures $90 \%$ power at a two-sided level of significance of 5\% (NCSS and PASS 11 (NCSS Statistical Software, Kaysville, UT, USA)). Assuming an expected withdrawal rate of $10 \%$ during the trial, 8 additional patients will be included and randomized; therefore, the total sample size required is $\mathrm{n}=88$ patients. Figure 1 .

\section{Eligibility criteria}

\section{Inclusion criteria}

Patients who meet the following criteria will be included in the study:

1. Patients suitable for undergoing both laparoscopic left hepatectomy and open left hepatectomy of the liver

2. Patients who be able to understand the nature of the study and what will be required of them

3. Men and nonpregnant, nonlactating women between the ages of 18 and 80

4. Patients with ASA I-II-III.

\section{Exclusion criteria}

Patients who meet any of the following criteria will be excluded from the study:

1. Inability to provide informed consent

2. Patients associated with a tumor or who require bilioenteric anastomosis or left caudate lobectomy

3. Immunodeficiency disease, such as HIV

4. Previous upper abdominal surgery (except for laparoscopic and open cholecystectomy).

\section{Withdrawal}

Patients can withdraw from the trial at any time at their own request or at the request of their legal representative. Patients may be removed if, in the researchers' opinion, continuing the trial may be detrimental to the patient's health, if left hepatectomy is not performed due to a technical inability, or for other reasons. Every withdrawal will be recorded in the clinical report forms (CRFs) and in the patient's medical case records. All examinations scheduled for the final trial day will be performed on all patients and documented. All dates will be analyzed according to the intention-to-treat (ITT) principle[24]. 


\section{Ethics, study registration, and consent}

The study has been approved by the Ethics Committee of the Second Affiliated Hospital of Anhui Medical University (identifier: KY20190809). The trial protocol has also been registered in the protocol registration system at ClinicalTrials.gov (identifier: NCT03958825). Patients willing to participate in this trial will receive both verbal and written information at the time of recruitment in the outpatient clinic of the Second Affiliated Hospital of Anhui Medical University. All participants of the study will be provided a participant information sheet and informed consent form entailing sufficient information. The participants will sign the general consent for the further use of personal data and biologic material. The investigators affirm and uphold the principle of the participant's right to privacy and that they shall comply with applicable privacy laws and/or the corresponding section of the study specific consent. The study procedure, benefits, risks, and data management will be clarified in detail during the preoperative conversation. All trial data will be saved and stored on a server during the trial, and patients will be asked to agree to future analysis.

\section{Trial interventions}

\section{The procedures of $L L H$}

The LLH operative procedures will be completed by the same surgical team in each group led by two experienced boardcertified surgeons. LLH and left lateral segmentectomy will be performed with the patient in the French position, with two experienced surgeons at each side (bilateral two chief surgeons) and a video laparoscope operator between the legs, which is different from the classic laparoscopic technique. Laparoscopic operations will be performed using 5 trocars. Common bile duct exploration (CBDE) will be performed in all patients with suspected stones in a dilated common bile duct (>10 mm) using a choledochoscope, followed by T-tube placement if postoperative cholangiography is required[12]. Figure 3.

\section{The procedures of $\mathrm{OLH}$}

OLH will be performed under general anesthesia with the patient in the supine position. An inverted L-shaped incision will be performed. The left hepatic artery and left portal vein will be individually dissected, ligated, and divided. Then, the left bile duct and left hepatic vein will be ligated, divided, and closed after hepatic parenchymal resection. The CBDE will be the same as the LLH.

\section{Primary and secondary endpoints Primary endpoint}

The LOS is designated as the primary outcome of this trial. Only the researchers and surgical surgeons will know the true operation. A blinded ward doctor will decide whether the patient should be discharged.

\section{Secondary endpoints}

The secondary outcomes are biliary leak[19], hemorrhage[20], post-hepatectomy liver failure[21], wound infection, intraabdominal fluid collection or abscess, relaparotomy, mortality, long-term abdominal wall hernias, hospital costs, residual stone rate and recurrence of calculus[22]. Existent ISGLS definitions will be used to ensure the comparability and generalizability of the results. Postoperative complications will be graded based on severity according to the Clavien-Dindo definition[25]. Table 1 and Table 2. 
Table 1

Definition of the endpoints

\begin{tabular}{|c|c|}
\hline Endpoints & Definition and assessment of outcomes \\
\hline $\begin{array}{l}\text { postoperative } \\
\text { length of } \\
\text { hospital stay }\end{array}$ & Time from the day of the operation until discharge (days) \\
\hline Operative time & Time from the beginning to end of the operation \\
\hline Mortality & Death due to any cause until 90 days after the operation and the reason \\
\hline Morbidity & $\begin{array}{l}\text { Postoperative complications will be recorded until } 90 \text { days after the operation. The severity of } \\
\text { complications will be graded according to the Clavien-Dindo classification }{ }^{[25]}\end{array}$ \\
\hline Blood loss & Total blood loss during the operation \\
\hline $\begin{array}{l}\text { Intraoperative } \\
\text { blood } \\
\text { transfusion }\end{array}$ & Massive hemorrhage with a hemoglobin level $<7 \mathrm{~g} / \mathrm{dl}$ or $\mathrm{Hct}<22$ \\
\hline $\begin{array}{l}\text { postoperative } \\
\text { length of } \\
\text { hospital stay }\end{array}$ & Time from the day of the operation until discharge (days) \\
\hline $\begin{array}{l}\text { Post- } \\
\text { hepatectomy } \\
\text { hemorrhage }\end{array}$ & $\begin{array}{l}\text { Evidence of blood loss from drains and/or nasogastric tube based on ultrasonography, as defined by } \\
\text { ISGPS (grade A, B or C) }{ }^{[20]}\end{array}$ \\
\hline Biliary leak & $\begin{array}{l}\text { Bilirubin concentration in the drain fluid at least three times the serum bilirubin concentration, as } \\
\text { defined by ISGLS (grade A, B or C) }{ }^{[19]}\end{array}$ \\
\hline $\begin{array}{l}\text { Post- } \\
\text { hepatectomy } \\
\text { liver failure }\end{array}$ & $\begin{array}{l}\text { The impaired ability of the liver to maintain its synthetic, excretory, and detoxifying functions, which } \\
\text { are characterized by an increased international normalized ratio and concomitant hyperbilirubinemia } \\
\text { (according to the normal }\end{array}$ \\
\hline & limits of the local laboratory) on or after postoperative day 5 , as defined by ISGLS (grade A, B or C) ) $^{[21]}$ \\
\hline $\begin{array}{l}\text { Intraabdominal } \\
\text { fluid collection }\end{array}$ & Collection of fluid measuring $\geq 3 \mathrm{~cm}$ associated with clinical or laboratory abnormalities \\
\hline Pneumonia & $\begin{array}{l}\text { Presence of a new infiltrate on the chest X-ray as well as the following: body temperature } ₫ 38^{\circ} \mathrm{C} \text {, } \\
\text { abnormal elevation of WBC, or positive sputum, and requiring antibiotic treatment }\end{array}$ \\
\hline $\begin{array}{l}\text { Wound } \\
\text { infection }\end{array}$ & Surgical site infection associated with laparotomy that develops during the initial hospital stay \\
\hline $\begin{array}{l}\text { Abdominal } \\
\text { rupture }\end{array}$ & $\begin{array}{l}\text { Dehiscence of abnormal closure with the need for resuture of the laparotomy during the initial hospital } \\
\text { stay }\end{array}$ \\
\hline $\begin{array}{l}\text { Total } \\
\text { hospitalization } \\
\text { expenses }\end{array}$ & Hospital costs from admission to discharge $(\$)$ \\
\hline $\begin{array}{l}\text { Recurrence of } \\
\text { calculus }\end{array}$ & $\begin{array}{l}\text { Calculi in the intrahepatic duct within } 3 \text { months after hepatectomy are } \\
\text { defined as residual stones }{ }^{[22]}\end{array}$ \\
\hline
\end{tabular}


Table 2

Severity grade according to the Clavien-Dindo definition[25]

\begin{tabular}{|c|c|}
\hline Grade & Definition \\
\hline ( & $\begin{array}{l}\text { Any deviation from the normal postoperative course without the need for pharmacological treatment or surgical, } \\
\text { endoscopic, and radiological intervention. }\end{array}$ \\
\hline ] & Requiring pharmacological treatment with drugs other than those allowed for grade $₫$ complications \\
\hline ( & Requiring surgical, endoscopic or radiological intervention \\
\hline 『a & Intervention not under general anesthesia \\
\hline$\nabla \mathrm{b}$ & Intervention under general anesthesia \\
\hline ( & Lifer-threatening complication. Requiring intensive care unit management \\
\hline 『a & Single-organ dysfunction \\
\hline$\nabla \mathrm{b}$ & Multiorgan dysfunction \\
\hline ૫ & Death of patient \\
\hline
\end{tabular}

Table 3

Schedule of enrollment, interventions, and assessments

\begin{tabular}{|c|c|c|c|c|c|c|c|c|c|c|}
\hline \multicolumn{11}{|l|}{ Screening } \\
\hline & $\begin{array}{l}\text { before } \\
\text { surgery }\end{array}$ & surgery & POD1 & POD2 & POD3 & Discharge & POD30 & $\begin{array}{l}6 \\
\text { months }\end{array}$ & $\begin{array}{l}12 \\
\text { months }\end{array}$ & $\begin{array}{l}24 \\
\text { months }\end{array}$ \\
\hline & & & & & & & & Postop & Postop & Postop \\
\hline $\begin{array}{l}\text { Selection } \\
\text { criteria and } \\
\text { informed } \\
\text { consent }\end{array}$ & $x$ & & & & & & & & & \\
\hline $\begin{array}{l}\text { Medical } \\
\text { history } \\
\text { Demographics }\end{array}$ & $x$ & & & & & & & & & \\
\hline $\begin{array}{l}\text { Physical } \\
\text { examination }\end{array}$ & $x$ & & & & & & & & & \\
\hline $\begin{array}{l}\text { Laboratory } \\
\text { texts }\end{array}$ & $x$ & & $x$ & $x$ & $x$ & $x$ & $x$ & $x$ & $x$ & $x$ \\
\hline $\begin{array}{l}\text { Trial } \\
\text { intervention }\end{array}$ & & $x$ & & & & & & & & \\
\hline $\begin{array}{l}\text { Intraoperative } \\
\text { outcomes }\end{array}$ & & $x$ & & & & & & & & \\
\hline $\begin{array}{l}\text { Postoperative } \\
\text { outcomes }\end{array}$ & & & $x$ & $x$ & $x$ & $x$ & $x$ & $x$ & $x$ & $x$ \\
\hline
\end{tabular}

\section{Type of trial}

The trial is a prospective randomized interventional single-center study comparing two parallel groups with a prospective registry to determine whether patients underwent LLS by laparoscopic surgery rather than open surgery with an enhanced recovery program. The types of interventions by patients, nurses, and ward doctors (not the surgeons performing the operations) before postoperative day (POD) 3 will be blinded. However, patients who are randomized to receive open or 
laparoscopic liver resection are at risk, as explained earlier. In addition, there is another potential source of bias when randomly selecting patients with strong treatment preferences. When patients are not informed about their treatment allocation (POD 3), they may be resentful and feel demoralized if they do not receive their preferred treatment. Thus, their compliance may be poor. By contrast, patients receiving their preferred treatment may have above-average compliance. Thus, to capitalize on centers both with and without preference for laparoscopic liver surgery and to acquire an uninterrupted prospective series of patients, all nonrandomized patients undergoing LLS will be approached to participate in the prospective registry. Registration of these patients is imperative to guarantee a consecutive series of patients and also because the absence of such a series may restrict the generalization of the results, as randomized participants may not, in fact, be representative[26]. The combination of an RCT and a prospective registry will improve the overall power and strengthen the external validity and generalizability of the study results[15, 27, 28].

\section{Randomization}

The randomization sequence is performed with sealed opaque envelopes. Upon completion of the screening assessment, participants who satisfy all the inclusion criteria and none of the exclusion criteria will be randomly assigned to the intervention and control groups in a 1:1 allocation ratio. Randomisation assignment will be computer generated using the 'randomize' library of SPSS22.0 (SPSS, Chicago, USA), with a complete randomising method based on permutations that guarantees balanced groups. A research nurse who will not participate in patient care will perform the randomization. Study group allocation will depend on the contents of sealed opaque envelopes generated in the above-described manner, and envelopes will be opened before operation.

\section{Blinding}

The patients and outcome observers will be blinded before POD 3 with respect to the trial intervention. Blinding of the surgeons and people involved in the operation is not feasible due to the nature of the interventions.

\section{Participating surgeons}

The surgical experience of surgeons can affect complication rates, which might bias the results. To prevent surgeon bias, surgeons should meet the following criteria: (1) have performed more than 200 open hepatectomies, (2) have performed more than 100 laparoscopic hepatectomies, and (3) be a qualified surgeon according to the China College of Laparoscopic Hepatectomy. LLH will be performed by two surgeons (Hui Hou and CL Wu), and OLH will be performed by three surgeons (Hui Hou, XP Geng and QR Xiong).

\section{Data management and quality assurance}

Data is collected prospectively for all patients including history, physical examination, laboratory data, pathologic examination, perioperative clinical information, and complications. Data is collected via datasheets on paper and kept securely. Study subjects will complete the examinations at inclusion, 1 weeks after surgery, and3, 6, 12, and 24months postoperatively. Follow-up will be followed up to 24 months postoperatively (Table 1). All handling cases are managed by subject identification code or anonymized registration number. The correspondence table of the anonymizing code and names and the consent form containing the names are kept strictly in the separate lockable document storage. All required parameters are collected in an SPSS data file (SPSS version 25, IBM statistics, Chicago, IL, USA).

\section{ERAS-Program}

All patients will participate in the ERAS liver program with a standardized peri-operative management. For the daily guidelines on the pre- and postoperative care of patients undergoing liver resection, see Fig. 2.

\section{Follow-up}

Follow-up is conducted at 1 month postoperatively and then $3,6,12$, and 24 months postoperatively by telephone or as outpatients with liver function test and abdominal ultrasonography for 2 years or until death. CT or MRCP is performed in 
cases of suspected stone recurrence or cholangitis.

\section{Statistical analysis}

The two-sided null hypothesis for the primary endpoint states that both study interventions will lead to a similar time to the LOS; the alternative hypothesis is that one intervention will perform better than the other. The null hypothesis will be tested by analyzing the covariance while adjusting for age and the type of liver resection. A binary logistic regression will be applied to compare the time to functional recovery between groups after adjusting for other factors. Background characteristics and surgical outcome measures will be compared using chi-squared or Fisher's exact tests for categorical data and two-tailed ttests or nonparametric Mann-Whitney $U$ tests for continuous variables. Categorical data will be presented as frequencies and group percentages, and continuous variables will be expressed as means and standard deviations. The homogeneity of the two groups will be described by comparison of the demographic data and baseline values. All analyses will be performed on an ITT basis [17]. For the ITT analyses, the data will be processed for all trial patients in their randomized groups. A P-value < 0.05 will be considered statistically significant. All statistical calculations will be performed using SPSS22.0 (SPSS version 25, IBM statistics, Chicago, IL, USA).

\section{Discussion}

The left side of the liver is a common site for and more seriously affected by hepatolithiasis. Hepatectomy has been the most effective treatment for removing IHD stones and resolving bile duct strictures, thereby decreasing the risk of recurrence[3, 22]. Compared with open hepatectomy, laparoscopic hepatectomy enables more accurate visualization and vascular anatomy. However, only a few retrospective studies comparing OLH and LLH without the ERAS program have been published summarizing their complications and long-term follow-up dates, such as residual stones and risk of recurrence, which are the main concerns of surgeons[11, 12].

The present study is the first RCT study to compare the benefits of the laparoscopic approach to left-side hepatolithiasis in Chinese patients undergoing hepatectomy with the ERAS program. It is well-recognized that a well-conducted double-blind RCT provides the highest level of evidence to prove the possible benefits of laparoscopic liver resection. However, performing an RCT during surgery is not without difficulties, and alternative trial designs may be necessary[23, 28]. First, the intervention needs to be tested in a standardized environment, and the properties of the intervention should remain unchanged during the trial period. The surgeons should have advanced laparoscopic experience and open liver resection experience. Both LLS and the ERAS program-enhanced recovery protocol provide the standardization needed. Second, the intervention should be double-blinded. Although double blinding in a surgical trial is difficult, using a fixed abdominal dressing for 3 days after surgery is feasible and should prevent both ward caregivers and patients from knowing the type of intervention. The trial with randomization of patients and surgeons with treatment equipoise and a prospective registry to cover both surgeons who believe that, based on their laparoscopic experience, randomization is not ethically justified and patients with a strong treatment preference will provide external validity. This trial design, which capitalizes on rather than ignores the differences between patients, will provide more robust outcome data and should lead to continuous performance monitoring after the trial.

Worldwide, the median LOS at a hospital for open and laparoscopic resections varies from 4 to 8 days[6, 9, 12]. The reasons for delay of discharge and the discharge location are often absent, and to date, a clear definition of recovery has only been used in a few publications. Departing from the standpoint that an RCT should be conducted, a primary question concerns which sample size should be used. In our opinion, a reduction of only 1.3 days in the time to recovery or LOS at a hospital after laparoscopic resection would be a disappointingly low gain. To prove such a reduction, 88 patients are needed ( $a=0.05$ and power of $90 \%$ ), making the trial unlikely to be reasonably moderate, and it is to be expected that patient accrual will be accomplished within 2-3 years.

This study has several limitations that should be acknowledged. It will be conducted at a single institution. Due to the small sample size, the findings from this trial will not allow for established clinical application but rather will serve to inform the 
need for larger multicenter RCTs on LLH for OLH.

\section{Abbreviations}

ASA, American Society of Anesthesiologists; CRFs, Clinical Report Forms; ISGLS, International Study Group of Liver Surgery;

\section{Declarations}

\section{Acknowledgements}

We thank QiaoHong Yang of the Department of Epidemiology and Health Statistics, Anhui Medical University, Hefei, for planning the statistical analyses. We are indebted to all patients who support this scientific research without personal benefit.

\section{Publication policies}

The sponsor will make every endeavor to publish the data in a medical journal(s) to communicate the results to healthcare professionals, the public, and other relevant groups. All participants will be sent a free copy of the published article(s). There will not be any publication restriction, and we plan to submit at least three major publications. We will also present preliminary results at national, regional, and international scientific meetings. The main investigators-Dr. Shubo Pan, Dr.Chunli Wu, and Prof. Hui Hou-will be either first or last authors in at least two of the three major publications. All investigators indicated in this protocol, and eventually additional colleagues participating in the future, will be coauthors of this study according to their individual contributions.

\section{Trial status}

This protocol represents the trial protocol version 1.0. The study is currently in the process of recruiting participants. Recruitment of participants commenced on 10 $0^{\text {th }}$ August 2019, and will be completed in December 2022.

\section{Author's contributions}

SBP conceived the study, drafted the manuscript, and revised the manuscript. XPG conceived the study and critically revised the manuscript. DCZ and HH calculated the sample size. SBP and ZDC drafted the manuscript. CLW and SBP drafted the study protocol. SBP and CLW revised the manuscript. All the authors read and approved the final manuscript.

\section{Authors' information}

All authors work at the Second Affiliated Hospital of Anhui Medical University, Furong Road 678\#, Shushan District, Hefei, Anhui 230601, China.

\section{Funding}

This study was supported by Clinical Research Cultivation Program of The Second Affiliated Hospital of Anhui Medical University(no:2020LCYB15).

\section{Availability of data and materials}

The datasets used and/or analyzed during the current study are available from the corresponding author on reasonable request.

\section{Ethics approval and consent to participate}


The study protocol was submitted for approval to the Ethics Committee of the Second Affiliated Hospital of Anhui Medical University (identifier: KY20190809). We distributed a written consent form to the participating patients and also informed them about the trial orally.

\section{Consent for publication}

Not applicable.

\section{Competing interests}

The authors declare that no competing interests exist.

\section{References}

1. Yang T, Lau WY, Lai EC, et al. Hepatectomy for bilateral primary hepatolithiasis: a cohort study[J]. Ann Surg. 2010;251(1):84-90.

2. Lee SE, Jang JY, Lee JM, et al. Selection of appropriate liver resection in left hepatolithiasis based on anatomic and clinical study[J]. World J Surg. 2008;32(3):413-8.

3. Cheung MT, Kwok PC. Liver resection for intrahepatic stones[J]. Arch Surg. 2005;140(10):993-7.

4. Yoon YS, Han HS, Shin SH, et al. Laparoscopic treatment for intrahepatic duct stones in the era of laparoscopy: laparoscopic intrahepatic duct exploration and laparoscopic hepatectomy[J]. Ann Surg. 2009;249(2):286-91.

5. Cai XJ, Yang J, Yu H, et al. Clinical study of laparoscopic versus open hepatectomy for malignant liver tumors[J]. Surg Endosc. 2008;22(11):2350-6.

6. Shin YC, Jang JY, Kang MJ, et al. Comparison of laparoscopic versus open left-sided hepatectomy for intrahepatic duct stones[J]. Surg Endosc. 2016;30(1):259-65.

7. Buell JF, Cherqui D, Geller DA, et al. The international position on laparoscopic liver surgery: The Louisville Statement, 2008[J]. Ann Surg. 2009;250(5):825-30.

8. Huang ZQ, Huang XQ, Zhang WZ, et al. [Liver resection in hepatolithiasis: 20-year's evolution][J]. Zhonghua Wai Ke Za Zhi. 2008;46(19):1450-2.

9. Peng JX, Wang LZ, Diao JF, et al. Major hepatectomy for primary hepatolithiasis: a comparative study of laparoscopic versus open treatment[J]. Surg Endosc. 2018;32(10):4271-6.

10. Im C, Cho JY, Han HS, et al. Validation of difficulty scoring system for laparoscopic liver resection in patients who underwent laparoscopic left lateral sectionectomy[J]. Surg Endosc. 2017;31(1):430-6.

11. Peng L, Xiao J, Liu Z, et al. Laparoscopic left-sided hepatectomy for the treatment of hepatolithiasis: A comparative study with open approach[J]. Int J Surg. 2017;40:117-23.

12. Chen S, Huang L, Qiu FN, et al. Total laparoscopic partial hepatectomy versus open partial hepatectomy for primary leftsided hepatolithiasis: A propensity, long-term follow-up analysis at a single center[J]. Surgery. 2018;163(4):714-20.

13. Tufo A, Dunne D, Manu N, et al. Hepatectomy for octogenarians with colorectal liver metastasis in the era of enhanced recovery[J]. Eur J Surg Oncol. 2018;44(7):1040-7.

14. Hughes MJ, Chong J, Harrison E, et al. Short-term outcomes after liver resection for malignant and benign disease in the age of ERAS[J]. HPB (Oxford). 2016;18(2):177-82.

15. Wong-Lun-Hing EM, van Dam RM, van Breukelen GJ, et al. Randomized clinical trial of open versus laparoscopic left lateral hepatic sectionectomy within an enhanced recovery after surgery programme (ORANGE II study) [J]. Br J Surg. 2017;104(5):525-35.

16. Liang $\mathrm{X}$, Ying $\mathrm{H}$, Wang $\mathrm{H}$, et al. Enhanced recovery care versus traditional care after laparoscopic liver resections: a randomized controlled trial[J]. Surg Endosc. 2018;32(6):2746-57. 
17. Stoot JH, van Dam RM, Busch OR, et al. The effect of a multimodal fast-track programme on outcomes in laparoscopic liver surgery: a multicentre pilot study[J]. HPB (Oxford), 2009,11(2):140-144.

18. McCulloch P. Developing appropriate methodology for the study of surgical techniques[J]. J R Soc Med. 2009;102(2):51-5.

19. Koch M, Garden OJ, Padbury R, et al. Bile leakage after hepatobiliary and pancreatic surgery: a definition and grading of severity by the International Study Group of Liver Surgery[J]. Surgery. 2011;149(5):680-8.

20. Rahbari NN, Garden OJ, Padbury R, et al. Post-hepatectomy haemorrhage: a definition and grading by the International Study Group of Liver Surgery (ISGLS)[J]. HPB (Oxford). 2011;13(8):528-35.

21. Rahbari NN, Garden OJ, Padbury R, et al. Posthepatectomy liver failure: a definition and grading by the International Study Group of Liver Surgery (ISGLS)[J]. Surgery. 2011;149(5):713-24.

22. Lee TY, Chen YL, Chang HC, et al. Outcomes of hepatectomy for hepatolithiasis[J]. World J Surg. 2007;31(3):479-82.

23. Ding G, Cai W, Qin M. Pure Laparoscopic Versus Open Liver Resection in Treatment of Hepatolithiasis Within the Left Lobes: A Randomized Trial Study[J]. Surg Laparosc Endosc Percutan Tech. 2015;25(5):392-4.

24. Montedori A, Bonacini MI, Casazza G, et al. Modified versus standard intention-to-treat reporting: are there differences in methodological quality, sponsorship, and findings in randomized trials? A cross-sectional study[J]. Trials. 2011;12:58.

25. Dindo D, Demartines N, Clavien PA. Classification of surgical complications: a new proposal with evaluation in a cohort of 6336 patients and results of a survey[J]. Ann Surg. 2004;240(2):205-13.

26. Nguyen KT, Gamblin TC, Geller DA. World review of laparoscopic liver resection-2,804 patients[J]. Ann Surg. 2009;250(5):831-41.

27. McCulloch P. Developing appropriate methodology for the study of surgical techniques[J]. J R Soc Med. 2009;102(2):51-5.

28. Gore SM. The consumer principle of randomisation[J]. Lancet. 1994;343(8888):58.

\section{Figures}




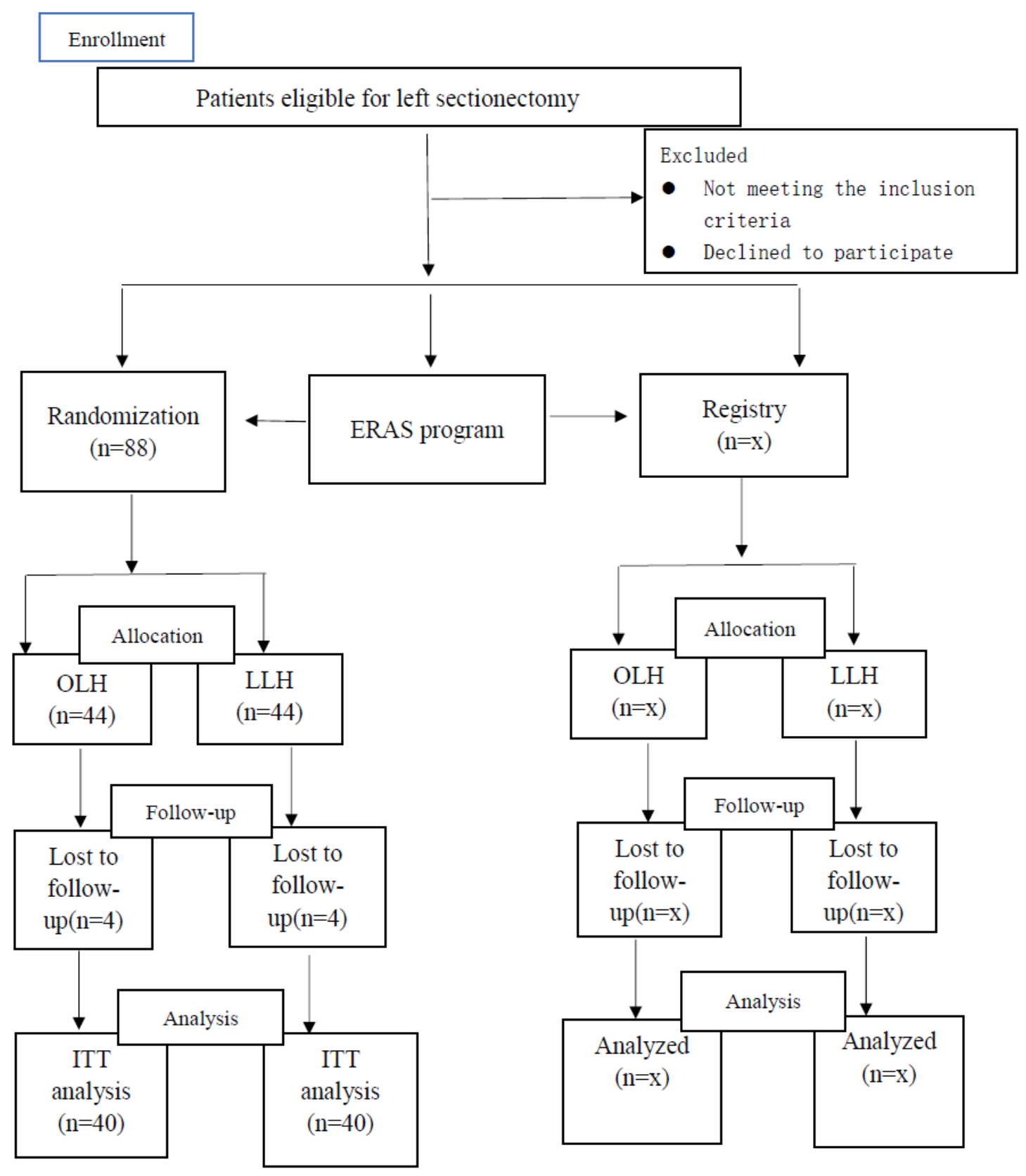

Figure 1

Flow chart according to CONSORT 
Day before surgery

Normal oral nutrition up to 6 hours before surgery

No bowel preparation

Laboratory tests

\section{Day of operation}

No pre-anesthetic medication

Carbohydrate $250 \mathrm{ml}$ drinks up to 2 hours

before surgery

No nasogastric tube

Transabdominal transverse fasciae plane

block + intravenous analgesia

Body air-warming device

I.V fluids warmed

goal-directed fluid therapy and avoid

excessive IV fluids (CVP during

transection $<5 \mathrm{mmHg}$ )

Patient sent to recovery ward

Restart oral intake of water

\section{Postoperative day (POD) 1}

Patients sent to surgical ward

Patient mobilizes a minimum of four times

a day

Discontinuation of IV fluids

Patient drinks at least 1.5 liters
Artificial intelligence patient-controlled analgesia (PCA) + Parecoxib 40mg IV

q12h to control pain

Remove urinary catheter

Laboratory tests

\section{POD 2}

Continue PCA or with Parecoxib 40mg IV q12h

Continue mobilization a minimum of four times a day

Normal diet

Laxatives

\section{POD3}

Stop PCA and Parecoxib

Start NSAID'S, such as celecoxibkai,

orally

Continue mobilization

Normal diet

Laxatives

Laboratory tests

Check discharge criteria

Outpatient appointment made on

postoperative day 7,12 or 14

\section{POD 4}

Check the discharge criteria

Discharge

Figure 2

ERAS program 


\section{Image not available with this version}

\section{Figure 3}

Surgical techniques for LLH

\section{Supplementary Files}

This is a list of supplementary files associated with this preprint. Click to download.

- SPIRITFillablechecklistSPIRIT2013Checklist.doc 